SECTION 7. Mechanics and machine construction.

Alexandr Petrovich Kerzhaev

Candidate of Phys. \& Math., Head of Laboratory of Department Computer Science and Computer Engineering, I. Yakovlev Chuvash State Pedagogical University, Cheboksary alex_kerg@mail.ru

Vyacheslav Gennadyevich Efremov Candidate of Phys. \& Math., Dean of Physical \& Mathematical Faculty, I. Yakovlev Chuvash State Pedagogical University, Cheboksary s1270266@mail.ru

Tatyana Valeryevna Mitrofanova

Candidate of Phys. \& Math., Assoc. Professor of Department Computer Science and Computer Engineering, I. Yakovlev Chuvash State Pedagogical University, Cheboksary mitrofanova tv@mail.ru

Tatyana Nikolaevna Pavlova

Candidate of Phys. \& Math., Assoc. Professor of Department Computer Science and Computer Engineering, I. Yakovlev Chuvash State Pedagogical University, Cheboksary tn_pavlova@mail.ru

\title{
STRAIN STATE OF A TWO-LAYER THICK-WALLED PIPE AT TRANSMITTING ANISOTROPY
}

Abstract: In this article the strain state of the two-layer thick-walled pipe being under the influence of internal pressure is considered. The material is supposed elastoplastic, each layer possesses the properties of transmitting anisotropy. Solution of the problem is the small parameter method. In the first approximation moving components in elastic and plastic areas are defined.

Keywords: moving, elasticity, plasticity, transmitting anisotropy, pipe, layer.

\section{ДЕФОРМИРОВАННОЕ СОСТОЯНИЕ ДВУХСЛОЙНОЙ ТОЛСТОСТЕННОЙ ТРУБЫ ПРИ ТРАНСЛЯЦИОННОЙ АНИЗОТРОПИИ}

Аннотация: В данной статье рассматривается деформированное состояние двухслойной толстостенной трубы, находящейся под действием внутреннего давления. Материал предполагается упруго-идеальнопластическим, каждый слой обладает своими свойствами трансляционной анизотропии. Решение задачи находится методом малого параметра. $B$ первом приближении определень компоненты перемещения в упругой и пластической областях.

Ключевые слова: перемещение, упругость, пластичность, транслячионная анизотропия, труба, слой.

Рассмотрим деформированное состояние двухслойной толстостенной трубы, соответствующей условиям предельного состояния

$$
\begin{gathered}
\left(\frac{\sigma_{\rho n}-\sigma_{\theta n}}{2}\right)^{2}+\tau_{\rho \theta n}^{2}-2 R_{n}\left(\frac{\sigma_{\rho n}-\sigma_{\theta n}}{2}\right) \cos \left(2 \theta+\mu_{n}\right)- \\
-2 \tau_{\rho \theta n} R_{n} \sin \left(2 \theta+\mu_{n}\right)+R_{n}^{2}-K_{n}^{2}=0,
\end{gathered}
$$

где

$$
R_{n}=\sqrt{\left(\frac{k_{1 n}-k_{2 n}}{2}\right)^{2}+k_{3 n}^{2}}, \frac{k_{1 n}-k_{2 n}}{2 R_{n}}=\cos \mu_{n}, \frac{k_{3 n}}{R_{n}}=\sin \mu_{n}, n=1,2
$$


Будем считать материал несжимаемым, коэффициент Пуассона $\mu=\frac{1}{2}$.

Согласно $[2,6]$ определим компоненты перемещения в нулевом приближении в упругой области

$$
\begin{aligned}
u_{2}^{(0) e}= & \frac{1}{2\left(\beta^{2}-1\right) E}\left[\left(q-2 K_{1} \ln \frac{\alpha_{2}}{\alpha_{1}}-2 K_{2} \ln \frac{1}{\alpha_{2}}\right) \rho+\right. \\
& \left.+3\left(q-2 K_{1} \ln \frac{\alpha_{2}}{\alpha_{1}}-2 K_{2} \ln \frac{1}{\alpha_{2}}\right) \frac{\alpha_{3}^{2}}{\rho}\right] .
\end{aligned}
$$

Согласно [2] компоненты перемещения в первом приближении в упругой области определяются в виде

$$
\begin{aligned}
& u_{2}^{(I) e}=\frac{\beta}{E}\left[-2(1+\mu) \tilde{C}_{1} \frac{\rho}{\beta}+2(1+\mu) \tilde{C}_{2}\left(\frac{\rho}{\beta}\right)^{-3}-4 \mu \tilde{C}_{3}\left(\frac{\rho}{\beta}\right)^{3}+4 \tilde{C}_{4} \frac{\beta}{\rho}\right] \times \\
& \times\left(a_{2}^{\prime \prime} \cos 2 \theta+b_{2}^{\prime \prime} \sin 2 \theta\right)+ \\
& +\frac{\beta}{E}\left[-2(1+\mu) \bar{C}_{1} \frac{\rho}{\beta}+2(1+\mu) \bar{C}_{2}\left(\frac{\rho}{\beta}\right)^{-3}-4 \mu \bar{C}_{3}\left(\frac{\rho}{\beta}\right)^{3}+4 \bar{C}_{4} \frac{\beta}{\rho}\right] \times \\
& \times\left(-a_{2}^{\prime \prime \prime} \sin 2 \theta+b_{2}^{\prime \prime \prime} \cos 2 \theta\right), \\
& v_{2}^{(I) e}=\frac{\beta}{E}\left[2(1+\mu) \tilde{C}_{1} \frac{\rho}{\beta}+2(1+\mu) \tilde{C}_{2}\left(\frac{\rho}{\beta}\right)^{-3}+(6+2 \mu) \tilde{C}_{3}\left(\frac{\rho}{\beta}\right)^{3}+(-2+2 \mu) \tilde{C}_{4} \frac{\beta}{\rho}\right] \times \\
& \times\left(a_{2}^{\prime \prime} \sin 2 \theta-b_{2}^{\prime \prime} \cos 2 \theta\right)+ \\
& +\frac{\beta}{E}\left[2(1+\mu) \bar{C}_{1} \frac{\rho}{\beta}+2(1+\mu) \bar{C}_{2}\left(\frac{\rho}{\beta}\right)^{-3}+(6+2 \mu) \bar{C}_{3}\left(\frac{\rho}{\beta}\right)^{3}+\right. \\
& \left.+(-2+2 \mu) \bar{C}_{4} \frac{\beta}{\rho}\right]\left(a_{2}^{\prime \prime \prime} \cos 2 \theta+b_{2}^{\prime \prime \prime} \sin 2 \theta\right),
\end{aligned}
$$

где

$$
\begin{gathered}
\beta=\alpha_{3}, \tilde{C}_{1}=\frac{-1+2 \beta^{2}-\beta^{-4}}{2 N}, \tilde{C}_{2}=\frac{-3+2 \beta^{2}+\beta^{4}}{6 N} \beta^{-4}, \\
\tilde{C}_{3}=\frac{-3+2 \beta^{-2}+\beta^{-4}}{6 N} \beta^{2}, \tilde{C}_{4}=\frac{-1+2 \beta^{-2}-\beta^{4}}{2 N} \beta^{-2}, \\
\bar{C}_{1}=\frac{4-4 \beta^{2}}{4 N}, \bar{C}_{2}=\frac{4 \beta^{2}-4 \beta^{4}}{12 N} \beta^{-4}, \\
\bar{C}_{3}=\frac{3-4 \beta^{-2}+\beta^{-4}}{12 N} \beta^{2}, \bar{C}_{4}=\frac{-1+\beta^{4}}{2 N} \beta^{-2} .
\end{gathered}
$$

В пластической области из (1) и ассоциированного закона течения найдем

$$
\begin{gathered}
\varepsilon_{\rho n}^{p}=\lambda_{n}\left[\frac{\sigma_{\rho n}-\sigma_{\theta n}}{2}-R_{n}^{\prime} \cos \left(2 \theta+\mu_{n}\right)\right\rfloor, \\
\varepsilon_{\theta n}^{p}=-\varepsilon_{\rho n}^{p}, \\
\varepsilon_{\rho \theta n}^{p}=\lambda_{n}\left[\tau_{\rho \theta n}-R_{n}^{\prime} \sin \left(2 \theta+\mu_{n}\right)\right] .
\end{gathered}
$$


Исключая величину $\lambda_{n}$ из (4), получим

$$
\frac{\varepsilon_{\rho \theta n}}{\varepsilon_{\rho n}-\varepsilon_{\theta n}}=\frac{\tau_{\rho \theta n}-R_{n}^{\prime} \sin \left(2 \theta+\mu_{n}\right)}{\sigma_{\rho n}-\sigma_{\theta n}-2 R_{n}^{\prime} \cos \left(2 \theta+\mu_{n}\right)} \text {. }
$$

В нулевом приближении уравнение несжимаемости имеет вид

$$
\varepsilon_{\rho n}^{(0)}+\varepsilon_{\theta n}^{(0)}=0 .
$$

Из (6) следует

$$
\frac{d u_{n}^{(0)}}{d \rho}+\frac{u_{n}^{(0)}}{\rho}=0
$$

где $u^{(0)}$ - компонента перемещения вдоль радиуса $\rho$, компонента перемещения $v^{0}$ вдоль $\theta$ равна нулю: $v^{0}=0$.

Решение уравнения (7) имеет вид

$$
u_{n}^{(0)}=\frac{C_{n}}{\rho}, C_{n}-\text { const } .
$$

Из условий сопряжения на границе слоев трубы

$$
u_{n}^{(0)}=u_{n+1}^{(0)} \text { при } \rho=\alpha_{n}
$$

и (8) найдем

$$
C_{n}=C_{n+1}
$$

Перепишем выражение (8) в виде

$$
u^{(0)}=\frac{C}{\rho}, C-\text { const },
$$

справедливо для всех слоев трубы.

Из (11) получим

$$
\varepsilon_{\rho}^{(0)}=\frac{d u^{(0)}}{d \rho}=-\frac{C}{\rho^{2}}, \quad \varepsilon_{\theta}^{0}=\frac{u^{(0)}}{\rho}=\frac{C}{\rho^{2}} .
$$

Положим, что $\tau_{\rho \theta n}^{(0)}=\varepsilon_{\rho \theta n}^{(0)}=0$.

Из (2), (11) получим компоненты перемещения во второй пластической области

$$
u_{2}^{(0) p}=\frac{C}{\rho}, v_{2}^{(0) p}=0,
$$

где

$$
C=\frac{1}{2\left(\beta^{2}-1\right) E}\left[\left(q-2 K_{1} \ln \frac{\alpha_{2}}{\alpha_{1}}-2 K_{2} \ln \frac{1}{\alpha_{2}}\right)+3\left(q-2 K_{1} \ln \frac{\alpha_{2}}{\alpha_{1}}-2 K_{2} \ln \frac{1}{\alpha_{2}}\right) \alpha_{3}^{2}\right] .
$$

Согласно (11) в первой пластической области компоненты перемещений имеют вид

$$
u_{1}^{(0) p}=\frac{C}{\rho}, v_{1}^{(0) p}=0 .
$$

Для определения компонент перемещений в первом приближении используем уравнение несжимаемости

$$
\varepsilon_{\rho n}^{(I)}+\varepsilon_{\theta n}^{(I)}=0, \varepsilon_{\rho n}^{(I)}=\frac{\partial u_{n}^{(I)}}{\partial \rho}, \quad \varepsilon_{\theta n}^{(I)}=\frac{u_{n}^{(I)}}{\rho}+\frac{1}{\rho} \frac{\partial v_{n}^{(I)}}{\partial \theta},
$$

откуда

$$
\frac{\partial u_{n}^{(I)}}{\partial \rho}+\frac{u_{n}^{(I)}}{\rho}+\frac{1}{\rho} \frac{\partial v_{n}^{(I)}}{\partial \theta}=0
$$


где $u_{n}^{(I)}, v_{n}^{(I)}$ - компоненты перемещения в первом приближении в $n$-ом слое.

Из (5) получим линеаризированное соотношение

$$
\left(\sigma_{\rho n}^{(0)}-\sigma_{\theta n}^{(0)}\right) \varepsilon_{\rho \theta n}^{(I)}=\left(\varepsilon_{\rho}^{(0)}-\varepsilon_{\theta}^{(0)}\right)\left[\tau_{\rho \theta n}^{(I)}-R_{n}^{\prime} \sin \left(2 \theta+\mu_{n}\right)\right] .
$$

Имеет место

$$
\varepsilon_{\rho \theta n}^{(I)}=\frac{1}{2}\left(\frac{\partial v_{n}^{(I)}}{\partial \rho}-\frac{v_{n}^{(I)}}{\rho}+\frac{1}{\rho} \frac{\partial u_{n}^{(I)}}{\partial \theta}\right) .
$$

Согласно (12), (17) уравнение (18) примет вид

$$
\begin{gathered}
\frac{\partial v_{n}^{(I)}}{\partial \rho}-\frac{v_{n}^{(I)}}{\rho}+\frac{1}{\rho} \frac{\partial u_{n}^{(I)}}{\partial \theta}= \\
=\frac{2 C}{K_{n} \rho^{2}} R_{n}^{\prime}\left\{\frac{\alpha_{1}}{\rho}\left(\cos \left(\sqrt{3} \ln \frac{\rho}{\alpha_{1}}\right)-\sqrt{3} \sin \left(\sqrt{3} \ln \frac{\rho}{\alpha_{1}}\right)\right)-2\right\} \sin \left(2 \theta+\mu_{n}\right) .
\end{gathered}
$$

Удовлетворим уравнение несжимаемости (16), полагая

$$
u_{n}^{(I)}=-\frac{1}{\rho} \frac{\partial \psi_{n}^{\prime}}{\partial \theta}, v_{n}^{(I)}=\frac{\partial \psi_{n}^{\prime}}{\partial \rho} .
$$

Из (19), (20) получим

$$
\begin{gathered}
\rho^{2} \frac{\partial^{2} \psi_{n}^{(1)}}{\partial \rho^{2}}-\rho \frac{\psi_{n}^{(1)}}{\partial \rho}-\frac{\partial^{2} \psi_{n}^{\prime}}{\partial \theta^{2}}= \\
=\frac{2 C}{K_{n}} R_{n}^{\prime}\left\{\frac{\alpha_{1}}{\rho}\left(\cos \left(\sqrt{3} \ln \frac{\rho}{\alpha_{1}}\right)-\sqrt{3} \sin \left(\sqrt{3} \ln \frac{\rho}{\alpha_{1}}\right)\right)-2\right\} \sin \left(2 \theta+\mu_{n}\right) .
\end{gathered}
$$

Решение уравнения (21) представим как сумму решений общего решения однородного уравнения и частного решения неоднородного уравнения. Частное решение неоднородного уравнения (21) будем искать в виде

$$
\begin{gathered}
\psi_{n \text { частн }}^{\prime}=\left(\frac{1}{\rho}\left[S_{n} \sin \omega+T_{n} \cos \omega\right]+Q_{n}\right) \sin \left(2 \theta+\mu_{n}\right), \\
\omega=\sqrt{3} \ln \frac{\rho}{\alpha_{1}} .
\end{gathered}
$$

Из (19), (22) найдем

$$
D_{1 n}=\frac{2 C}{K_{n}} R_{n}^{\prime}, S_{n}=-\frac{\sqrt{3} \alpha_{1} D_{1 n}}{8}, T_{n}=-\frac{\alpha_{1} D_{1 n}}{8}, Q_{n}=\frac{-D_{1 n}}{2} .
$$

Из (20), (21) получим

$$
\begin{gathered}
u_{n \text { частн }}^{(I)}=-\frac{2}{\rho^{2}}\left(\left[S_{n} \sin \left(\sqrt{3} \ln \frac{\rho}{\alpha_{1}}\right)+T_{n} \cos \left(\sqrt{3} \ln \frac{\rho}{\alpha_{1}}\right)\right]+Q_{n} \rho\right) \cos \left(2 \theta+\mu_{n}\right), \\
v_{n \text { частн }}^{(I)}=-\frac{1}{\rho^{2}}\left[\left(S_{n}+T_{n} \sqrt{3}\right) \sin \left(\sqrt{3} \ln \frac{\rho}{\alpha_{1}}\right)+\left(T_{n}-S_{n} \sqrt{3}\right) \cos \left(\sqrt{3} \ln \frac{\rho}{\alpha_{1}}\right)\right] \sin \left(2 \theta+\mu_{n}\right) .
\end{gathered}
$$

Согласно (20), компоненты перемещений в пластической области, соответствующие решению однородного уравнения (21) имеют вид 


$$
\begin{aligned}
u_{n \text { одम }}^{(I) p}= & -2\left\{\left[C_{11}^{(n)} \cos (\sqrt{3} \ln \rho)+C_{12}^{(n)} \sin (\sqrt{3} \ln \rho)\right] \sin 2 \theta-\right. \\
- & {\left.\left[C_{21}^{(n)} \cos (\sqrt{3} \ln \rho)+C_{22}^{(n)} \sin (\sqrt{3} \ln \rho)\right] \cos 2 \theta\right\}, } \\
& v_{n \text { oдн }}^{(I) p}=\left[C_{11}^{(n)}(\cos (\sqrt{3} \ln \rho)-\sqrt{3} \sin (\sqrt{3} \ln \rho))+\right. \\
+ & \left.C_{12}^{(n)}(\sin (\sqrt{3} \ln \rho))+\sqrt{3} \cos (\sqrt{3} \ln \rho)\right] \sin 2 \theta+ \\
& +\left[C_{21}^{(n)}(\cos (\sqrt{3} \ln \rho)-\sqrt{3} \sin (\sqrt{3} \ln \rho))+\right. \\
+ & \left.C_{22}^{(n)}(\sin (\sqrt{3} \ln \rho))+\sqrt{3} \cos (\sqrt{3} \ln \rho)\right] \cos 2 \theta .
\end{aligned}
$$

На упругопластической границе имеют место условия сопряжения

$$
u_{2}^{(I) p}=u_{2}^{(I) e}, v_{2}^{(I) p}=v_{2}^{(I) e} \text { при } \rho=1 .
$$

Из (3), (24)-(26) получим

$$
\begin{gathered}
C_{11}^{(2)}=-\frac{B_{1}}{2}+\sin \mu_{2}\left(S_{2} \sin \omega_{1}+T_{2} \cos \omega_{1}+Q_{2}\right), \\
C_{12}^{(2)}=\frac{\sqrt{3}}{6}\left(B_{1}-2 \sin \mu_{2}\left(S_{2} \sin \omega_{1}+T_{2} \cos \omega_{1}+Q_{2}\right)+\right. \\
\left.+2 \cos \mu_{2}\left(S_{2} \sin \omega_{1}+T_{2} \cos \omega_{1}-S_{2} \sqrt{3} \cos \omega_{1}+T_{2} \sqrt{3} \sin \omega_{1}\right)+2 B_{2}\right), \\
C_{21}^{(2)}=\frac{A_{1}}{2}+\cos \mu_{2}\left(S_{2} \sin \omega_{1}+T_{2} \cos \omega_{1}+Q_{2}\right), \\
C_{12}^{(2)}=-\frac{\sqrt{3}}{6}\left(A_{1}+2 \cos \mu_{2}\left(S_{2} \sin \omega_{1}+T_{2} \cos \omega_{1}+Q_{2}\right)-\right. \\
\left.-2 \sin \mu_{2}\left(S_{2} \sin \omega_{1}+T_{2} \cos \omega_{1}-S_{2} \sqrt{3} \cos \omega_{1}-T_{2} \sqrt{3} \sin \omega_{1}\right)-2 A_{2}\right),
\end{gathered}
$$

где

$$
\begin{gathered}
\omega_{1}=\sqrt{3} \ln \frac{1}{\alpha_{1}}, \\
A_{1}=\frac{\beta a_{2}^{\prime \prime}}{E}\left[-2(1+\mu) \tilde{C}_{1} \beta^{-1}+2(1+\mu) \tilde{C}_{2} \beta^{3}-4 \mu \tilde{C}_{3} \beta^{-3}+4 \tilde{C}_{4} \beta\right]+ \\
+\frac{\beta b_{2}^{\prime \prime \prime}}{E}\left[-2(1+\mu) \bar{C}_{1} \beta^{-1}+2(1+\mu) \bar{C}_{2} \beta^{3}-4 \mu \bar{C}_{3} \beta^{-3}+4 \bar{C}_{4} \beta\right], \\
B_{1}=\frac{\beta b_{2}^{\prime \prime}}{E}\left[-2(1+\mu) \tilde{C}_{1} \beta^{-1}+2(1+\mu) \tilde{C}_{2} \beta^{3}-4 \mu \tilde{C}_{3} \beta^{-3}+4 \tilde{C}_{4} \beta\right]- \\
-\frac{\beta a_{2}^{\prime \prime \prime}}{E}\left[-2(1+\mu) \bar{C}_{1} \beta^{-1}+2(1+\mu) \bar{C}_{2} \beta^{3}-4 \mu \bar{C}_{3} \beta^{-3}+4 \bar{C}_{4} \beta\right], \\
A_{2}=-\frac{\beta b_{2}^{\prime \prime}}{E}\left[2(1+\mu) \tilde{C}_{1} \beta^{-1}+2(1+\mu) \tilde{C}_{2} \beta^{3}+(6+2 \mu) \tilde{C}_{3} \beta^{-3}+(-2+2 \mu) \tilde{C}_{4} \beta\right]+ \\
+\frac{\beta a_{2}^{\prime \prime \prime}}{E}\left[2(1+\mu) \bar{C}_{1} \beta^{-1}+2(1+\mu) \bar{C}_{2} \beta^{3}+(6+2 \mu) \bar{C}_{3} \beta^{-3}+(-2+2 \mu) \bar{C}_{4} \beta\right],
\end{gathered}
$$




$$
\begin{gathered}
B_{2}=\frac{\beta a_{2}^{\prime \prime}}{E}\left[2(1+\mu) \tilde{C}_{1} \beta^{-1}+2(1+\mu) \tilde{C}_{2} \beta^{3}+(6+2 \mu) \tilde{C}_{3} \beta^{-3}+(-2+2 \mu) \tilde{C}_{4} \beta\right]+ \\
+\frac{\beta b_{2}^{\prime \prime \prime}}{E}\left[2(1+\mu) \bar{C}_{1} \beta^{-1}+2(1+\mu) \bar{C}_{2} \beta^{3}+(6+2 \mu) \bar{C}_{3} \beta^{-3}+(-2+2 \mu) \bar{C}_{4} \frac{\beta}{\rho}\right] .
\end{gathered}
$$

Согласно (3), (24)-(26) компоненты перемещений в первом приближении во втором слое определены.

Условия сопряжения компонент перемещений на границе слоев трубы имеют вид

$$
u_{2}^{(I) p}=u_{1}^{(I) p}, v_{2}^{(I) p}=v_{1}^{(I) p} \text { при } \rho=\alpha_{2} .
$$

Из (24)-(28) определим коэффициенты $C_{11}^{(1)}, C_{12}^{(1)}, C_{21}^{(1)}, C_{22}^{(1)}$

$$
\begin{gathered}
C_{11}^{(1)}=\frac{\sqrt{3}}{6}\left(\sin \omega_{2}\left(H_{1}-N_{1}+2 H_{2}-2 N_{2}\right)+\sqrt{3} \cos \omega_{2}\left(H_{1}-N_{1}\right)\right), \\
C_{12}^{(1)}=-\frac{\sqrt{3}}{6}\left(\sqrt{3} \sin \omega_{2}\left(-H_{1}+N_{1}\right)+\sqrt{3} \cos \omega_{2}\left(H_{1}-N_{1}+2 H_{2}-2 N_{2}\right)\right), \\
C_{21}^{(1)}=-\frac{\sqrt{3}}{6}\left(\sin \omega_{2}\left(L_{1}-M_{1}+2 M_{2}-2 L_{2}\right)+\sqrt{3} \cos \omega_{2}\left(L_{1}-M_{1}\right)\right), \\
C_{22}^{(1)}=\frac{\sqrt{3}}{6}\left(\sqrt{3} \sin \omega_{2}\left(-L_{1}+M_{1}\right)+\sqrt{3} \cos \omega_{2}\left(L_{1}-M_{1}+2 M_{2}-2 L_{2}\right)\right),
\end{gathered}
$$

где

$$
\begin{gathered}
M_{1}=2 C_{21}^{(2)} \cos \omega_{2}+2 C_{22}^{(2)} \sin \omega_{2}-2 \cos \mu_{2} \frac{S_{2} \sin \omega_{3}+T_{2} \cos \omega_{3}+Q_{2} \alpha_{2}}{\alpha_{2}^{2}}, \\
N_{1}=-2 C_{11}^{(2)} \cos \omega_{2}-2 C_{12}^{(2)} \sin \omega_{2}+2 \sin \mu_{2} \frac{S_{2} \sin \omega_{3}+T_{2} \cos \omega_{3}+Q_{2} \alpha_{2}}{\alpha_{2}^{2}}, \\
M_{2}=C_{21}^{(2)} \cos \omega_{2}-\sqrt{3} \sin \omega_{2}+C_{22}^{(2)} \sin \omega_{2}+\sqrt{3} \cos \omega_{2}- \\
-\sin \mu_{2} \frac{S_{2} \sin \omega_{3}+T_{2} \cos \omega_{3}-S_{2} \sqrt{3} \cos \omega_{3}+T_{2} \sqrt{3} \sin \omega_{3}}{\alpha_{2}^{2}}, \\
N_{2}=C_{11}^{(2)} \cos \omega_{2}-\sqrt{3} \sin \omega_{2}+C_{12}^{(2)} \sin \omega_{2}+\sqrt{3} \cos \omega_{2}- \\
-\cos \mu_{2} \frac{S_{2} \sin \omega_{3}+T_{2} \cos \omega_{3}-S_{2} \sqrt{3} \cos \omega_{3}+T_{2} \sqrt{3} \sin \omega_{3}}{\alpha_{2}^{2}}, \\
L_{1}=-2 \cos \mu_{1} \frac{S_{1} \sin \omega_{3}+T_{1} \cos \omega_{3}+Q_{1} \alpha_{2}}{\alpha_{2}^{2}}, H_{1}=2 \sin \mu_{1} \frac{S_{1} \sin \omega_{3}+T_{1} \cos \omega_{3}+Q_{1} \alpha_{2}}{\alpha_{2}^{2}}, \\
L_{2}=-\sin \mu_{1} \frac{S_{1} \sin \omega_{3}+T_{1} \cos \omega_{3}-S_{1} \sqrt{3} \cos \omega_{3}+T_{1} \sqrt{3} \sin \omega_{3}}{\alpha_{2}^{2}}, \\
H_{2}=-\cos \mu_{1} \frac{S_{1} \sin \omega_{3}+T_{1} \cos \omega_{3}-S_{1} \sqrt{3} \cos \omega_{3}+T_{1} \sqrt{3} \sin \omega_{3}}{\alpha_{2}^{2}} \\
\omega_{2}=\sqrt{3} \ln \alpha_{2}, \omega_{3}=\sqrt{3} \ln \frac{\alpha_{2}}{\alpha_{1}} .
\end{gathered}
$$

Согласно (24), (25), (28), (29) компоненты перемещений в первом слое в первом приближении определены. 


\section{References:}

1. Биценко К. Б. Техническая динамика / К. Б. Биценко, Р. Граммель. - Л. : Гостехиздат, 1950. - $900 \mathrm{c.}$

2. Ивлев Д. Д. Метод возмущений в теории упругопластического тела / Д. Д. Ивлев, Л. В. Ершов. - М. : Наука, 1978. - 208 с.

3. Ивлев Д. А. О предельном состоянии слоистых круговых цилиндров из анизотропного материала под действием внутреннего давления / Д. А. Ивлев // Вестник Чувашского государственного педагогического университета им. И. Я. Яковлева. - 2010. - № 2 (66). - С. 57-63.

4. Кержаев А. П. Деформированное состояние анизотропного кольцевого диска, находящегося под действием равномерных растягивающих усилий / А. П. Кержаев // Наука и образование в XXI веке : сб. науч. тр. по материалам междунар. науч.практ. конф. : в 6 ч. Ч. ІІІ. - М. : АР-Консалт, 2013. - С. 139-140.

5. Кержаев А. П. Деформированное состояние анизотропной тонкой пластины при двуосном растяжении / А.П. Кержаев // Международное научное издание «Современные фундаментальные и прикладные исследования» / International scientific periodical «Modern fundamental and applied researches». - Кисловодск, 2013. - № 1 (8). - T. 1. - C. 84-87.

6. Кержаев А. П. Упругопластическое состояние двухслойной толстостенной трубы, находящейся под действием внутреннего давления, в случае трансляционной анизотропии / А. П. Кержаев // Вестник Чувашского государственного педагогического университета им. И. Я. Яковлева. Серия : Механика предельного состояния. - 2013. - № 2 (16). - С. 54-61.

7. Никитин А. В. Определение деформированного состояния толстостенной нелинейно-неоднородной трубы при трансляционной анизотропии / А. В. Никитин // Вестник Чувашского государственного педагогического университета им. И. Я. Яковлева. Серия : Механика предельного состояния. - 2014. - № 1 (19). С. $60-70$.

8. Митрофанова Т. В. Напряженно-деформированное упругопластическое состояние тонкой анизотропной пластины, ослабленной круговым отверстием при двуосном растяжении / Т. В. Митрофанова, Т. Н. Павлова // Вестник Чувашского государственного педагогического университета им. И. Я. Яковлева. Серия : Механика предельного состояния. - 2013. - № 3 (17). - С. 105-110.

9. Павлова Т.Н. Об определении перемещений в задаче напряженнодеформированного состояния тонкой пластины с эллиптическим отверстием / Т. Н. Павлова // Вестник Чувашского государственного педагогического университета им. И. Я. Яковлева. Серия : Механика предельного состояния. -2010. - № 1 (65). - С. 64-69.

10. Фоминых С. О. Упругопластическое состояние толстостенной трубы при взаимодействии различных видов пластической анизотропии / С. О. Фоминых // Вестник Чувашского государственного педагогического университета им. И. Я. Яковлева. - 2011. - № 1 (9). - С. 201-216. 